\title{
EANM commitment towards involvement and engagement of patients and the public: learning from the UK experience
}

\author{
Ana Sofia Ribeiro ${ }^{1} \cdot$ Martin Lee $^{2} \cdot$ Wim J. G. Oyen ${ }^{2}$ (I)
}

Received: 11 July 2019 / Accepted: 22 July 2019 /Published online: 2 August 2019

(C) Springer-Verlag GmbH Germany, part of Springer Nature 2019

\begin{abstract}
"No matter how complicated the research, or how brilliant the researcher, patients and the public always offer unique, invaluable insights. Their advice when designing, implementing and evaluating research invariably makes studies more effective, more credible and often more cost efficient as well." Professor Dame Sally C. Davies, 2009 [1]
\end{abstract}

Commonly referred to as patient/public involvement and engagement (PPI/E) as well as user involvement or lay involvement, $\mathrm{PPI} / \mathrm{E}$ incorporates and integrates individual's perspectives in the planning and improvement of healthcare services and research $[2,3]$.

Also known as patient advocacy or patient input, $\mathrm{PPI} / \mathrm{E}$ is incorporated in daily clinical practice and throughout the different stages of research, reflecting issues that are important and relevant to those whom it potentially affects [4].

In 1996, the National Institute for Health Research (NIHR) in the UK established INVOLVE [5] which is now well rooted within research and strongly encourages PPI/E contributions, while even being mandatory when applying to funding bodies within the UK [4].

Across health and social care services, PPI/E ranges from management boards, commissioning boards, professional associations, amongst others and this reflects the acknowledgment that PPI/E is crucial to drive service improvement [3].

This article is part of the Topical Collection on Editorial

Wim J. G. Oyen

president@eanm.org

Royal Marsden NHS Foundation Trust, London, UK

2 The European Association of Nuclear Medicine, Vienna, Austria
Across Europe, PPI/E has been gaining global momentum in recent years $[6,7]$. PPI/E has been advocated by the European Lung Foundation [8] and together with NIHR, they developed tools for patients under the European Patient Ambassador Program (EPAP) to introduce patients to terminology and skills to work collaboratively with researchers and healthcare professionals [9]. Other initiatives include the European Patient's Academy (EUPATI) which developed a focused and intense training for patients on the process of medicine development [10] and a recent European project, Patients Active in Research and Dialogues for an Improved Generation of Medicines (PARADIGM), aims to engage and involve patients in strategic research processes within the life cycle of medicines [11].

The impact of PPI/E may even extend to patient peer review, whereby patients, patients' representatives and carers' are invited to form part of the peer review process, as introduced in 2014 by the British Medical Journal (BMJ).

In the UK, the NIHR recently published a research cycle model (Fig. 1) that captures the different stages of research where PPI/E can be involved. The model demonstrates that patients and public can be engaged from early phase research with identification of research themes right to dissemination of results to groups and forums and be co-authors in journal and newsletter articles. PPI/E members can establish links with key policy makers to support changes in practice and by recording the impact PPI/E on the study and publishing this alongside the main study findings provides support for researchers and future projects.

$\mathrm{PPI} / \mathrm{E}$ can help shape the research question and design, support the development of patient information sheets, consent forms and provide input on patient recruitment by assessing potential patient burden of the various nuclear medicine procedures. PPI/E work scope should also include data collection, analysis of data, attendance of steering group meetings and the dissemination of findings.

Nuclear medicine and the overall imaging community are part of the workforce who will ultimately translate science into 
impact

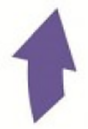

Implementing

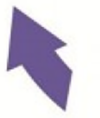

Disseminating

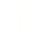

\section{Identifying and prioritising \\ $\checkmark$}
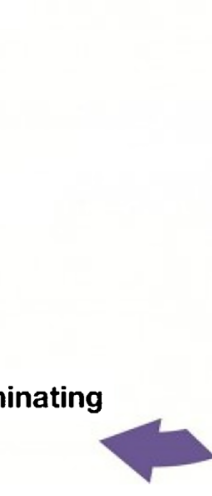

Undertaking

Fig. 1 The research cycle [4]

bedside clinical practice, but in order to increase the relevance, impact and overall quality, clinicians and researchers must work collaboratively with PPI/E [12].

$\mathrm{PPI} / \mathrm{E}$ is emerging as a priority in planning and delivering healthcare services, awareness campaigns and information material to patients and public, whereby the experiences and knowledge of users can be used to promote and benefit others leading to patientcentered care.

In nuclear medicine, PPI/E can be incorporated in the production of patient and public information, making valuable changes in the communication between healthcare professionals and patients, informing on access to services and leading on support groups for therapy patients.

There are a number of tools designed on how and when to integrate PPI/E into research studies and how PPI/E should be supported during a research study including specific training needs for PPI/E [4, 13] and these can also be applied to clinical practice.

Nuclear medicine and imaging research in general should consider a more structured, shared approach to the incorporation of PPI/E at all levels of research studies and should consider combining their efforts to raise awareness of PPI/E in nuclear medicine research.

However, the complexities of introducing PPI/E across Europe should not be underestimated. The EANM has 43 member states, providing a rich mix of cultures and a wide variety of healthcare systems. Therefore, delivering meaningful public involvement in nuclear medicine will require a common agenda and cross-border cooperation in establishing and sharing best practice.

\section{Compliance with ethical standards}

Conflict of interest The authors declare that they have no conflict of interest.

\section{References}

1. Staley K. Exploring impact: public involvement in NHS, public health and social care research. www.invo.org.uk/wp-content/uploads/2011/11/Involve_Exploring_Impactfinal28.10.09.pdf.

2. Mockford C, Staniszewska S, Griffiths F, Herron-Marx S. The impact of patient and public involvement on UK NHS health care: a systematic review. International Journal for Quality in Health Care : journal of the International Society for Quality in Health Care. International Journal for Quality in Health Care. 2012;24:28-38.

3. Lalani M, Baines R, Bryce M, Marshall M, Mead S, Barasi S, et al. Patient and public involvement in medical performance processes: a systematic review. Health Expectations : an international journal of public participation in health care and health policy. Health Expectations. 2019;22:149-161.

4. INVOLVE. INVOLVE: briefing notes for researchers: public involvement in NHS, public health and social care research. http:// www.invo.org.uk/wp-content/uploads/2014/11/9938_INVOLVE_ Briefing_Notes_WEB.pdf.

5. Research and Development Directorate DoH. Best research for best health. A new national health research strategy. http://assets. publishing.service.gov.uk/government/uploads/system/uploads/ attachment_data/file/568772/dh_4127152_v2.pdf.

6. Brett J, Staniszewska S, Mockford C, Herron-Marx S, Hughes J, Tysall C, et al. A systematic review of the impact of patient and public involvement on service users, researchers and communities. Patient. 2014;7:387-395.

7. Caron-Flinterman JF, Broerse JE, Teerling J, Bunders JF. Patients' priorities concerning health research: the case of asthma and COPD research in the Netherlands. Health Expectations. 2005;8:253-263.

8. European Lung Foundation: patient input 2013-2018 [updated 18/ 05/2018. Available from: http://www.europeanlung.org/en/getinvolved/patient-input/.

9. European Patient Ambassador Programme: the Programme 2013 [updated 18/05/2018. Available from: https://www.epaponline. org/what-is-epap/.

10. EUPATI. European Patients Academy: what is EUPATI? : EUPATI; 2018 [updated 30/05/2018. Available from: https://uk.eupati.eu/.

11. PARADIGM. PARADIGM: Our approach 2018 [updated 18/05/ 2018. Available from: http://imi-paradigm.eu/.

12. Chalmers JD, Timothy A, Polverino E, Almagro M, Ruddy T, Powell $\mathrm{P}$, et al. Patient participation in ERS guidelines and research projects: the EMBARC experience. Breathe (Sheff). 2017;13:194 207.

13. Mathie E, Wythe H, Munday D, Millac P, Rhodes G, Roberts N, et al. Reciprocal relationships and the importance of feedback in patient and public involvement: a mixed methods study. Health Expectations. 2018;21:899-908.

Publisher's note Springer Nature remains neutral with regard to jurisdictional claims in published maps and institutional affiliations. 